tensive adhesions. This lady died at the age of thirty-seven of cancer of the creum; the uterus was quite healthy.

These and other similar cases which I have met with prove that great care should be shown when this plan of treatment is followed, but not that it is one which should not be adopted if imperatively required. If the caustic is not too severely applied, on the one hand, and, on the other, the canal is kept open by passing a common bougie once or twice a week regularly, until the surface acted on is healed, and all tendency to contraction have ceased, no morbid diminution of the calibre of the cervical canal can ensue. Many of the cases which I see being extreme ones, I not unfrequently have to resort to this mode of treatment, and yet I have very seldom had occasion to dilate the cervical canal afterwards; and when I have, it has been because accidental circumstances have taken the patient out of my reach whilst under treatment. As a rule, I should say that no patient, in whose case the caustic potash has been applied to the cervical canal, should be lost sight of in less than six weeks, and during that time the canal should be kept open by the passage of a moderate-sized bougie once or twice a week.

The other accidents which may follow the use of caustic potash are, extension of the caustic to the vagina, and extension of the inflammatory reaction produced to the uterus and peritonæum. These accidents, like the former, may be avoided by common care and prudence. Potassa fusa itself ought, I think, to be discarded, now that we have in the potassa cum calce cylinders such an admirable and safe substitute. All the instances in which I have seen the vagina compromised have been cases in which pure potassa fusa had been used. It is so extremely deliquescent, that it is all but impossible to always avoid its running into the adjoining parts. As regards the extension of the secondary inflammation, that need not be feared if due precautions are taken both before and after the caustic is applied. All acute or even sub-acute inflammatory action should be first subdued, and the proper time should be chosen for the operation. Four or five days after menstruation is the best time, as it allows two or three weeks' quiescence from menstrual molimen. Lastly, the eschar produced should not be too extensive.

One of the chief arguments that hare been adduced against the use of caustic potash to the neck of the uterus is, that it produces cicatrices that may interfere with the process of parturition. This is merely a theoretical objection, not founded on observation, and devoid of truth. The fact is, that the faintest trace of even a deep eschar produced in this region, either by a caustic or by the actual cautery, ceases to be visible after the lapse of a few months. So far from causing induration, the action of these surgical agents is to melt and soften induration of the cervix when the latter is the result of chronic inflammation, as is usually the case, by favourably modifying the morbid nutrition of the parts diseased. The idea of hard cicatrices has been taken from the observation of what occurs in the skin, without taking into consideration that the structure of the skin and of mucous membrane is essentially different. In the skin there is a fully developed fibrous framework, which is the principal foundation of the hard cicatrix that follows any loss of substance in which it is involved. This fibrous framework is merely rudimentary in mucous membranes, and thence the facility with which any loss of substance in them is repaired. This we see exemplified in the mouth and intestinal canal, where all traces of ulcerative action are eventually lost. In the cervix uteri we see how nature repairs divisions and losses of substance, by observing what occurs after the lacerations of the substance of the cervix, which are so common in parturition, and which, when no subsequent inflammation sets up, merely leave a soft notch as the trace of their occurrence.

Such being the case, it is clear that the application of potassa fusa to the cervix uteri, so far from hardening the organ, and proving an impediment to future labours, acts in the reverse manner, positively facilitating parturition, by removing chronic inflammatory hypertrophy. Indeed, I may here remark, that the more I progress in life the more $I$ become convinced of the truth of an assertion which I made many years ago-viz., that most of the cases of rigid, undilating os uteri met with in practice are occasioned by chronic inflammatory disease of that organ, and not by constitutional conditions, spasms, \&c., an important fact in practical midwifery.

In concluding these remarks on the use and abuse of caustic potash in the treatment of inflammatory disease of the uterus, I wish to lay stress on the fact, that I merely recommend it, and resort to it, when there is actual disease present, when the cervix is the seat of chronic inflammatory action, intractable to all other agents, general and local, and when the hypertrophy is cansed and kept up by such disease. In those cases of hyper. trophy in which the cervix is merely passively enlarged, in which inflammatory action either does not exist or has given way to treatment or time, it ought not to be resorted to. The enlargement may then be safely left to nature and to general treatment. The absorbent powers of the uterus are, perhaps, greater than those of any other organ in the economy, and are generally sufficient, in the course of time, to fine down the enlarged cervix, when all actual disease has been removed.

I must be allowed to add, that the potassa cum calce cylinders constitute a very valuable and manageable caustic, whenever such an agent is required, for the destruction of chancres, the treatment of indolent sores, \&c. I have found it of great use in the treatment of hrmorrhoids, and, in some cases, preferable to the nitric acid, which has been of late so much recom. mended.

Grosvenor-street, 1854 .

\section{ON THE TREATMENT OF CHOLERA.}

\section{By W. WILKINSON, Esq., Sydenham.}

As cholera appears now unfortunately to have made its appearance once more amongst us, I would beg to offer some suggestions as to its treatment, which I hope may prove of some practical utility, and I do so with the less reserve, as there seems to be some variety of opinion upon the subject, and as there are few writers who appear to me to treat the subject in a sound common-sense manner. I would also beg leave to hint, with the greatest respect, that unless the treatment of this formidable disease, as well indeed as that of most others, be not based upon broad scientific principles, the vis medicatrix naturce had better, as a general rule, be allowed to work its own way.

Cholera may be divided into three stages-

The first, or preliminary stage, being diarrhoea, painless or otherwise.

The second, diarrhoa continued, motions extremely thin, and devoid. of fæeal smell, cramps, \&c.

The third being the stage of collapse, with unconsciousness, \&c.

The first stage may be generally relieved by the exhibition of a stimulant emetic, which, by evacuating the stomach, prevents any accumulation of irritative matter there, and by its revulsive power produces a general alterative effect. This is to be followed by the following pills:-Chloride of mercury, five grains; ipecacuanha powder, half a grain; soap and opium pill, ten grains: divided into three pills.

The calomel as well as the ipecacuanha to act upon the secretions, and thus relieve the bowel of any viscid irritative matter; the opium to relieve or prevent pain, nervous irritation, and excitement, (so constantly followed by depression;) and with the ipecacuanha to produce an effect upon the capillaries of the skin, and thus canse a sympathetic derivative action. This may be followed by an evacuant, consisting of rhubarb and magnesia, with compound cinnamon powder and water.

This, even in the early part of the second stage, will be suffi. cient sometimes to cure the patient; but if the serous fluil lost be considerable, or if the disease should run into its third stage, it is of importance to administer some astringent which can be depended upon, together with some medicine, so to uphold or stimulate the nervous power, as to prevent entire loss of control over the capillaries of the intestinal mucous membrane.

From my own experience in diarrhcea and some few cases of cholera, I can corroborate the opinions of several members of our profession as to the efficacy of sulphuric acid; therefore I should-in every case in the second stage, after the preliminary treatment, or immediately in the third stage, when, from the copious action of the bowels, the preliminary treatment wonld be unnecessary-preseribe, dilute sulphuric acid, half a fluid drachm; strychnia in acid phosphoric solution, one-sixteenth of a grain. This medicine to be taken as frequently as the urgency of the symptoms might render it necessary. The sulphuric acid may be depended on, I think, as an astringent, and the stychnia (as mentioned in a recent paper of mine, published in THE LAXNCE) would also be of the greatest benefit to uphold or stimulate the nervous power, and thus prevent that loss of control over the capillaries, which seems productive of such infinite mischief.

If on trial these suggestions should be of use to the profession in reducing the mortality from this formidable disease, my object is accomplished.

$J_{\text {tuly }}, 155$ t, 